OPEN ACCESS

Edited by:

Asma Bouden

Tunis El Manar University, Tunisia

Reviewed by:

Lara Rösler,

University of Würzburg, Germany Mercedes Gabriela Orozco Solis, University of Guadalajara, Mexico

*Correspondence: Miguel Castelo-Branco mcbranco@fmed.uc.pt

tThese authors have contributed equally to this work and share senior authorship

Specialty section:

This article was submitted to Social Cognition,

a section of the journa

Frontiers in Psychiatry

Received: 11 December 2020 Accepted: 02 February 2021

Published: 22 March 2021

Citation:

Mouga S, Castelhano J, Café C Sousa D, Duque F, Oliveira G and Castelo-Branco M (2021) Social Attention Deficits in Children With Autism Spectrum Disorder: Task Dependence of Objects vs. Faces

Observation Bias.

Front. Psychiatry 12:640599. doi: 10.3389/fpsyt.2021.640599

\section{Social Attention Deficits in Children With Autism Spectrum Disorder: Task Dependence of Objects vs. Faces Observation Bias}

\author{
Susana Mouga ${ }^{1,2,3,4}$, João Castelhano ${ }^{1,2}$, Cátia Café ${ }^{4}$, Daniela Sousa ${ }^{1,2,4}$, \\ Frederico Duque ${ }^{1,3,4,5,6}$, Guiomar Oliveira ${ }^{1,3,4,5,6+}$ and Miguel Castelo-Branco ${ }^{1,2,7 * t}$ \\ ${ }^{1}$ CIBIT - Coimbra Institute for Biomedical Imaging and Translational Research, University of Coimbra, Coimbra, Portugal, \\ ${ }^{2}$ ICNAS - Institute of Nuclear Sciences Applied to Health, University of Coimbra, Coimbra, Portugal, ${ }^{3}$ CNC.IBILI - Institute for \\ Biomedical Imaging and Life Sciences, Faculty of Medicine, University of Coimbra, Coimbra, Portugal, ${ }^{4}$ Neurodevelopmental \\ and Autism Unit From Child Developmental Centre, Hospital Pediátrico, Centro Hospitalar e Universitário de Coimbra, \\ Coimbra, Portugal, ${ }^{5}$ Centro de Investigação e Formação Clínica, Hospital Pediátrico, Centro Hospitalar e Universitário de \\ Coimbra, Coimbra, Portugal, ${ }^{6}$ University Clinic of Pediatrics, Faculty of Medicine, University of Coimbra, Coimbra, Portugal, \\ ${ }^{7}$ Faculty of Medicine, University of Coimbra, Coimbra, Portugal
}

Social attention deficits represent a central impairment of patients suffering from autism spectrum disorder (ASD), but the nature of such deficits remains controversial. We compared visual attention regarding social (faces) vs. non-social stimuli (objects), in an ecological diagnostic context, in 46 children and adolescents divided in two groups: ASD $(N=23)$ and typical neurodevelopment (TD) $(N=23)$, matched for chronological age and intellectual performance. Eye-tracking measures of visual scanning, while exploring and describing scenes from three different tasks from the Autism Diagnostic Observation Schedule (ADOS), were analyzed: "Description of a Picture," "Cartoons," and "Telling a Story from a Book." Our analyses revealed a three-way interaction between Group, Task, and Social vs. Object Stimuli. We found a striking main effect of group and a task dependence of attentional allocation: while the TD attended first and longer to faces, ASD participants became similar to TD when they were asked to look at pictures while telling a story. Our results suggest that social attention allocation is task dependent, raising the question whether spontaneous attention deficits can be rescued by guiding goal-directed actions.

Keywords: autism spectrum disorder, social attention, eye-tracking, attentional bias, autism diagnostic observation schedule

\section{INTRODUCTION}

Autism spectrum disorder (ASD) is an early-onset neurodevelopmental disorder marked by the specificity of significant impairments in social interaction and communication, restricted interests, and the presence of repetitive and stereotyped behaviors (1). Social deficits in other domains include deviations in basic attentional processes, impairments in attention to faces or social stimuli across the lifespan, as well as attention during social exchanges; for a review, see (2-5). 
The ability to direct the attention to social stimuli is present and evident in typically developing children from early infancy onwards (6-8). The attention to faces serves as a window into individuals' emotional and intentional states, providing critical information for social, cognitive, and communicative development and functioning (9-14). It has been hypothesized that deficits in social attention present in ASD, such as reduced attention to social stimuli as a whole or atypical allocation of attention to social stimuli, are the cause of a compromised social functioning. Such deficits might lead to reduced social processing, loss of information necessary for the development of adaptive social functioning $(15,16)$, and difficulties in the interpretation of emotional information $(17,18)$.

Eye-tracking studies in ASD showed a correlation between reduced attention to social stimuli and behavioral measures (1922). Klin et al. (21) showed, early on, that adolescents with ASD spent significantly less time attending to people when watching a segment of a movie and more time attending to the objects and the background of the scene. Deficits in social attention were thereafter replicated: (i) when looking at pictures of social scenes, participants with ASD spent less time attending to faces (23); (ii) ASD children showed no difference in the time looking at people or objects, unlike in typical neurodevelopment (TD) (24); and (iii) ASD attended less to the activities of others and focused more on the background objects (22).

Atypical imbalance in the attention for social (e.g., videos of playing children) vs. non-social stimuli (repeated geometrical patterns) in ASD was reported in a large-cohort study (25). A meta-analysis on gaze patterns (26) suggested the presence in ASD of a problem in selecting socially relevant vs. irrelevant information.

Other studies, however, do not confirm this hypothesis. Kemner et al. (27) found that the fixation times on face drawings embedded in an assortment of distractors of both children with ASD and TD were similar. Parish-Morris et al. (28) found that ASD and TD children and adolescents did not differ in the attention toward movies of faces as opposed to objects. In a study focused on magic, Kuhn et al. (29) found that ASD individuals were more susceptible to magic tricks, relying on sensitivity to social cues, than TD controls, contrary to their expectation. They found no between-group differences in fixation time on the magician's face and eyes. These studies suggest that the type of context and task may be relevant to disclose differences in social attentional allocation.

Several studies with infants suggest that innate or earlyemerging attentional biases for faces or complex social scenes may be intact within the first months of life in infants who later

\footnotetext{
Abbreviations: ADI-R, Autism Diagnostic Interview-Revised; ADOS, Autism Diagnostic Observation Schedule; AFD, Average Fixation Duration; ANOVA, analysis of variance; AOI, area of interest; ASD, autism spectrum disorder; CHUC, Centro Hospitalar E Universitário De Coimbra; CI, confidence interval; DSM, Diagnostic and Statistical Manual of Mental Disorders; DT\%, Dwell Time Percentage; ET, Entry Time; FFD, First Fixation Duration; FSIQ, Full-Scale Intelligence Quotient; FT\%, Fixation Time Percentage; IQ, Intelligence Quotient; MANOVA, multivariate analysis of variance; NDT\%, Net Dwell Time Percentage; NormD, Normalized Dwell; PIQ, Performance IQ; SD, standard deviation; TD, typical neurodevelopment.
}

develop $\operatorname{ASD}(30,31)$ in line with negative results from behavioral studies in early infancy (32).

Other important aspect to consider in the study of social attention with eye tracking in ASD is the ecological and task relevance of the stimuli. Static stimuli have been associated with no group differences, which might indicate that they are not optimally sensitive. Adding to this information, it has been suggested that scenes depicting ecological social interactions are the ones that evoke robust social responses (33-35).

So far, no consensus has been reached on whether social attention is fundamentally reduced or absent in individuals with ASD. We hypothesize that the role of type of stimuli and task is critical. This may explain why a large number of studies show significantly diminished attention to social information in ASD compared to TD controls $(21-23,36-39)$, while many others show no differences (27-29, 40-44). Given this discrepancy, it is important to understand whether the putative social attention deficits are task and stimuli dependent.

We previously showed that task and context are determinant for perceptual performance in ASD, only showing coherence loss in task conditions favoring local attentional analysis (45). The same might hold for the attentional bias that characterizes this population. In this study, we extended this prior work by comparing attention allocation to social vs. non-social stimuli in three tasks in ASD and TD children and adolescents. We used a paradigm based on stimuli from the Autism Diagnostic Observation Schedule (ADOS) (46), a diagnostic tool that we used as a routine in the diagnostic procedure, to try to see if it can discriminate between ASD and TD children in what concerns to visual social attention and corroborate the attentional bias claim. We hypothesized that ASD children differ from TD children in visual attention to social stimuli and, in particular, demonstrate less looking toward faces than TD children.

\section{METHODS \\ Participants}

The study comprised two groups of participants: the experimental, composed by individuals with ASD without intellectual disability (1), and the control, composed by individuals with typical neurodevelopment (TD). A total of 46 children and adolescents were enrolled in the study: 23 for the ASD group (22 male, 1 female; mean age $=13$ years and 1 month, minimum age $=8$ years and 10 months, maximum age $=17$ years and 4 months) and 23 for the control group (21 Male, 2 female; mean age $=13$ years and 5 months, minimum age $=8$ years and 5 months, maximum age $=17$ years and 8 months). Sample sizes were based on previously established effect sizes from other studies using eye tracking (23). Groups were matched by chronological age, gender, and performance intelligence quotient (PIQ) (47). Analyses were conducted to ensure that participants were matched with respect to chronological age, gender, handedness, and PIQ in both groups ( $T$-test, $p>0.05$ ). Further group characterization details can be found in Table 1.

ASD participants were recruited from a sample from the Neurodevelopmental and Autism Unit, Child Developmental Center, Pediatric Hospital, Centro Hospitalar e Universitário 
TABLE 1 | Characterization of the clinical and control groups.

\begin{tabular}{|c|c|c|c|}
\hline & ASD & TD & \\
\hline & Mean (SE) & Mean (SE) & $T$ tests \\
\hline N & 23 & 23 & \\
\hline Gender (M/F) & $22 / 1$ & $21 / 2$ & $p>0.05$ \\
\hline CA (months) & $156.8(4.9)$ & $160.5(6.4)$ & $p>0.05$ \\
\hline FSIQ & $99.2(3.0)$ & $124.8(4.1)$ & \\
\hline VIQ & $96.3(2.7)$ & $123.9(4.1)$ & \\
\hline$P I Q$ & 104.0 (3.2) & 112.1 (3.6) & $p>0.05$ \\
\hline ADI-R RSI & $15.7(1.1)$ & - & \\
\hline ADI-R L/C & $9.4(0.8)$ & - & \\
\hline ADI-R RB/I & $5.1(0.6)$ & - & \\
\hline ADOS COM & $4.6(0.3)$ & - & \\
\hline ADOS SI & $8.0(0.5)$ & - & \\
\hline ADOS Total & $12.6(0.7)$ & - & \\
\hline
\end{tabular}

ASD, Autism Spectrum Disorder group; TD, typical neurodevelopment group; SE, standard error of the mean; M, male; F, female; CA, chronological age; FSIQ, FullScale Intelligence quotient (IQ); VIQ, Verbal IQ; PIQ, Performance IQ; $A D I-R R S I$, $A D I-R$ Reciprocal Social Interactions; ADI-R L/C, Autism Diagnostic Interview-Revised Language/Communication; RB/I, ADI-R Repetitive Behaviors/Interests; ADOS COM, ADOS Communication; ADOS SI, ADOS Social Interaction. T-tests; $p>0.05$.

de Coimbra, Portugal. ASD diagnosis was assigned on the basis of the gold standard instruments: parental or caregiver interview, Autism Diagnostic Interview-Revised (ADI-R) (48); direct structured proband assessment, ADOS (46); and clinical examination performed by an experienced neurodevelopmental pediatrician, based on the current diagnostic criteria for autism spectrum disorder from the Diagnostic and Statistical Manual of Mental Disorders 5, DSM-5 (1). All ASD patients had positive results in the ADI-R and ADOS for autism or ASD and met the criteria for ASD from the DSM-5. A comprehensive medical observation excluded associated medical condition such as epilepsy, neurocutaneous or other genetic syndromes, or other usual comorbidities in ASD samples. TD participants were recruited from local schools and from our volunteers' database.

The exclusion criteria for the children who participated in this study were evaluated through an extensive anamnesis carried out with the parents or caregivers. They included neurological, neurodevelopmental, and genetic diseases, brain lesions, sensory, auditory, motor deficits, and neurodevelopmental milestones. Additionally, the parents of TD participants completed the Social Communication Questionnaire and Social Responsiveness Scale to ensure the exclusion of ASD symptomatology. TD participants with history of ASD symptomatology, other neurodevelopmental and neurological disorders, as well as other diseases mentioned above were excluded.

Both groups underwent an exhaustive neuropsychological evaluation and an assessment of the IQ to exclude intellectual disabilities [all participants had a Full-Scale IQ (FSIQ) > 70]. To be included in this study, participants also had to be able to read, describe pictures, and also remain still during the task. This increased the age of the participants able to be included, despite the efforts to recruit younger subjects.

\section{Apparatus}

Eye movements were measured with a remote binocular eye tracking (SMI RED) system (SMI-SensoMotoric Instruments, Germany), with a sampling rate of $250 \mathrm{~Hz}$. The tracker has a reported gaze position accuracy of $0.4^{\circ}$ and a spatial resolution of 0.05 . The participants sat $\sim$ between 60 and $70 \mathrm{~cm}$ away from a 22 -in flat screen with a resolution of $1,680 \times 1,050$ pixels. The system compensates for head movements within a $50 \mathrm{~cm} \times 30 \mathrm{~cm}$ (at $65 \mathrm{~cm}$ distance), allowing the participants to look at the screen in a naturalistic manner. A 9-point calibration procedure with a fixation cross was performed before each task. The children were instructed to fixate on the cross. After the calibration, there was a validation trial to ensure the precision of the data collection. The calibration process was repeated when necessary until both eyes achieved good mapping on all nine test positions (tracking error smaller than $1^{\circ}$ visual angle).

\section{Visual Stimuli and Task}

The experimental protocol was created and implemented through SensoMotoric Instruments Experiment Center Version 3.2 (SMI-SensoMotoric Instruments, Germany). It was composed of three types of tasks, which integrated visual stimuli adapted from the ADOS (46). The ADOS is a semistructured, examiner's dependent, tool to assess communication, social interaction, and imagination. It allows to diagnose autism spectrum disorder (ASD) across ages, neurodevelopmental levels, and language skills. The tasks from ADOS adapted were "Description of a Picture," "Cartoons," and "Telling a Story from a Book." In the "Description of a Picture" task, the participant was asked to look at a scene and tell what she/he sees. In the "Cartoons" and "Telling a story from a Book" tasks, they are asked to tell a story from the images that are presented one at a time as if they were really narrating a book. Therefore, they were instructed to tell the researcher when they want to move to the following page. In total, they were 25 visual stimuli, representing social scenes, displayed on a screen.

Participants were allowed to move forward to the next stimulus as soon as they had described what they were seeing and told the experimenter they wanted to move to the next picture. This choice was made instead of using a button to prevent subjects' fatigue, or boredom, and a subsequent attention decrease that may lead to just pressing the button without the description. To increase subjects' attention, involvement, and motivation, and to assure that the exploration of the image begins at the same point, between each image, a fixation cross was always presented. This fixation cross disappeared once the participant's gaze was detected to be within the embedded area of interest (AOI) in it. Figure 1 illustrates the experimental procedure. There was no time constraint in each picture.

\section{Eye Tracking Recordings and Analysis}

Eye movement data were recorded with iViewX ${ }^{\mathrm{TM}} 2.7$ and analyzed offline with BeGaze ${ }^{\mathrm{TM}} 3.7$ software where different AOIs were defined in a semiautomatic procedure: "faces" and "objects" (Figure 2).

We considered the following gaze metrics (defined in accordance with the manual of the BeGaze ${ }^{\mathrm{TM}} 3.7$ software): 


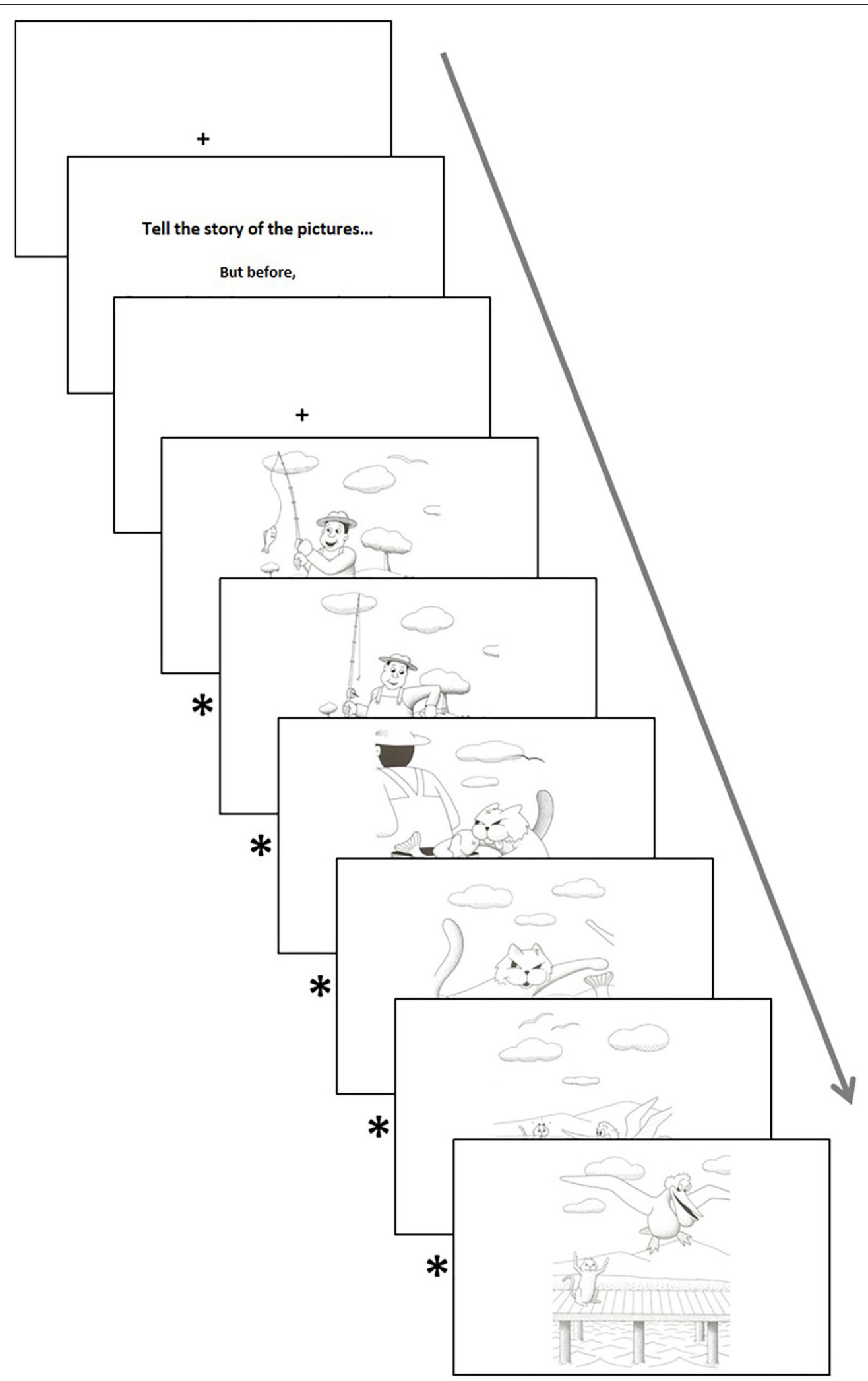

FIGURE 1 | Acquisition protocol of the task "Cartoons." *Between each image is always presented a fixation cross (signaled with an asterisk in the schematic representation of the task) to ensure that the exploration of the image begins at the same point. 

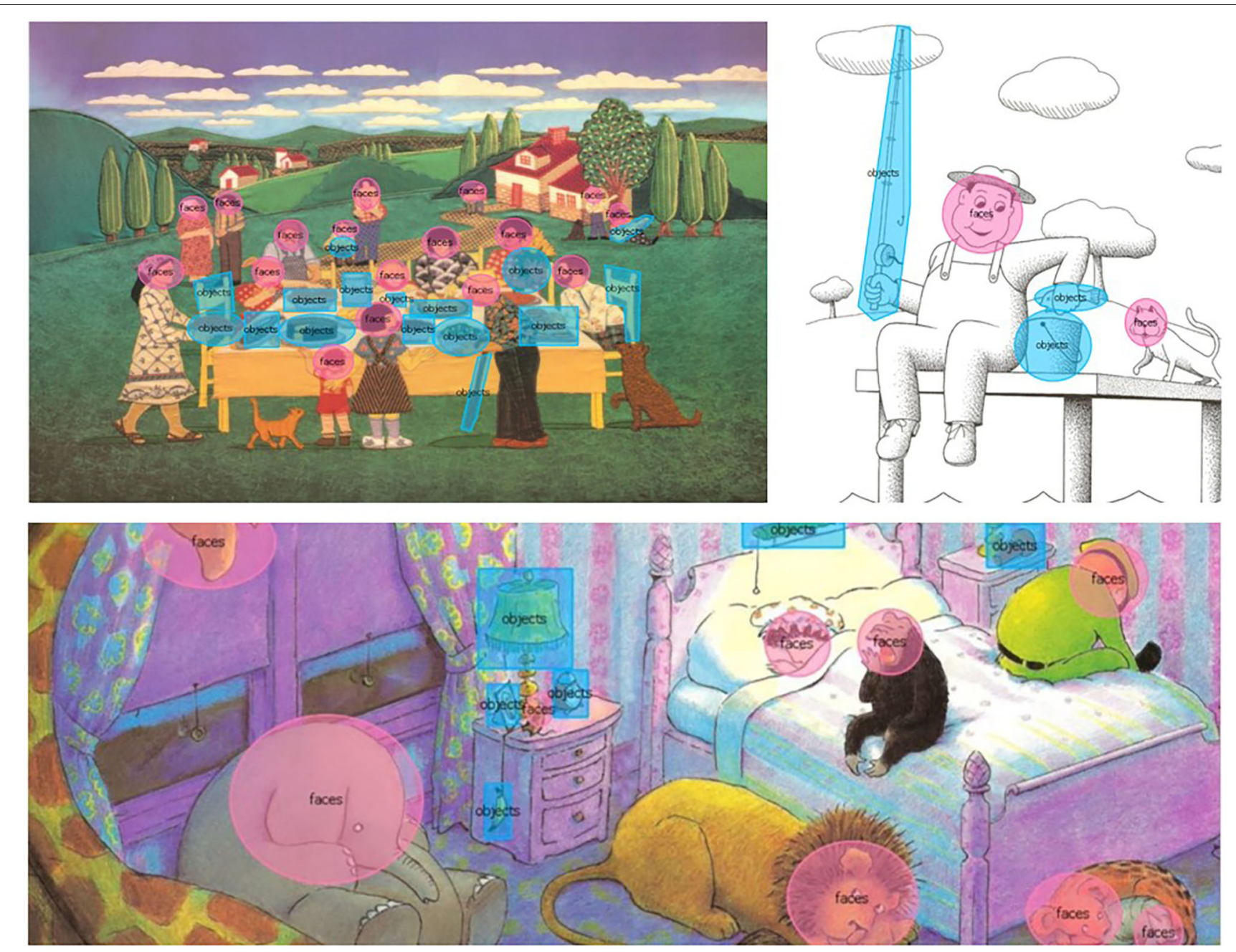

FIGURE 2 | Example of the different areas of interest defined.

Entry Time (ET), Dwell Time Percentage (DT\%), Net Dwell Time Percentage (NDT\%), Normalized Dwell (ms/coverage) (NormD), First Fixation Duration (FFD), Fixation Time Percentage (FT\%), Average Fixation Duration (AFD), and Revisits. Entry Time expresses the average interval in milliseconds ( $\mathrm{ms}$ ) from the presentation of the visual stimuli (start of the trial) to the first gaze fixation on each area of interest (AOI). The Dwell Time Percentage (DT\%) consists of the percentage of the sum of durations from all fixations and saccades (between the first and last fixations within the AOI) that hit the AOI (in ms), divided by the total stimulus duration. NDT\% represents the percentage of the sum of sample durations for all gaze data samples that hit the AOI, divided by the total stimulus duration. NormD is the ratio between the DT and the AOI coverage, where coverage is the AOI size (measured in pixel) in comparison to stimulus size, thus representing a percentage of the number of pixel $(\mathrm{px})$. It represents a more reliable measure to understand attention distribution patterns since it adjusts the duration that a subject spent to process an item relative to its surface in the display. FFD represents the duration (in ms) of the first fixation to hit the AOI. FT\% consists of the percentage of the sum of the fixation durations inside the AOI, divided by the total stimulus duration. AFD is the total duration (ms) of all fixations divided by the number of fixations inside the AOI. A longer fixation duration is often associated with a deeper and more effortful cognitive processing. Revisits represent the number of time subjects visit an AOI.

\section{Data Analysis and Statistics}

Initially, we conducted a descriptive statistics analysis in order to characterize the sample.

Eye-position data were analyzed with a standard AOI approach. Eye-tracking data were preprocessed using the SMI software, BeGaze Version 3.7 (SMI-SensoMotoric Instruments, Germany), which uses a dispersion-based algorithm for detecting fixations. The minimum fixation duration was $80 \mathrm{~ms}$, and the maximum dispersion value was 100 pixels. Different aspects of eye movements were assessed. We included seven dependent 
variables in our eye-tracking analyses: ET, NDT\%, NormD, FFD, FT\%, AFD, and Revisits.

One participant in the TD group was excluded from analysis due to problems with eye-tracking data collection in tasks Picture and Book. In total, there were, therefore, valid data for 23 ASD and 22 TD participants in the Picture and Book Tasks and for 23 ASD and 23 TD participants in the Cartoons Task.

Multivariate analysis of variance (MANOVA) with a threeway interaction was used to evaluate differences in the eyetracking measures by group, task, and AOI type (faces or objects). The goal of the three-way MANOVA was to understand if there was an interaction effect for group, AOI type, and task in the eye-tracking measures. Follow-up univariate three-way ANOVAs were run for each dependent variable. In the dependent variables with statistically significant interaction effects, simple two-way interactions and main effect of group were run. In the statistically significant main effect of group, pairwise comparisons were run with a Bonferroni adjustment applied. Effect sizes (partial $\eta^{2}$ for $F$ statistics and Cohen's $d$ for Bonferroni) are reported with $p$-values for significant main effects, interactions, and pairwise comparisons. Our MANOVA reached a power of $92 \%$.

All statistical analysis was completed with the support of the version for Microsoft Windows ${ }^{\circledR}$ of the Statistical Package for Social Sciences, version 26 (SPSS $^{\circledR}$, Chicago, IL, USA). A significance level of 0.05 was adopted.

\section{Ethics Statement}

All the procedures in this study were reviewed and approved by the ethics committees from the Faculty of Medicine from University of Coimbra, Portugal (CE-11/2013) and the Centro Hospitalar e Universitário de Coimbra, Portugal (CHUC-10213) and was conducted in accordance with the 1964 Helsinki declaration and its later amendments or comparable ethical standards. Written informed consent was obtained from the parents/guardians of all participants. Children and adolescents also gave oral informed consent.

\section{RESULTS}

A three-way MANOVA was run with seven eye-tracking measures (ET, NDT\%, NormD, FFD, FT\%, AFD, and Revisits) as dependent variables and independent variables: Group (ASD and TD), Task (Picture, Cartoons, and Book), and AOI (faces and objects). There was a statistically significant three-way interaction between Group, Task, and AOI in all our dependent variables

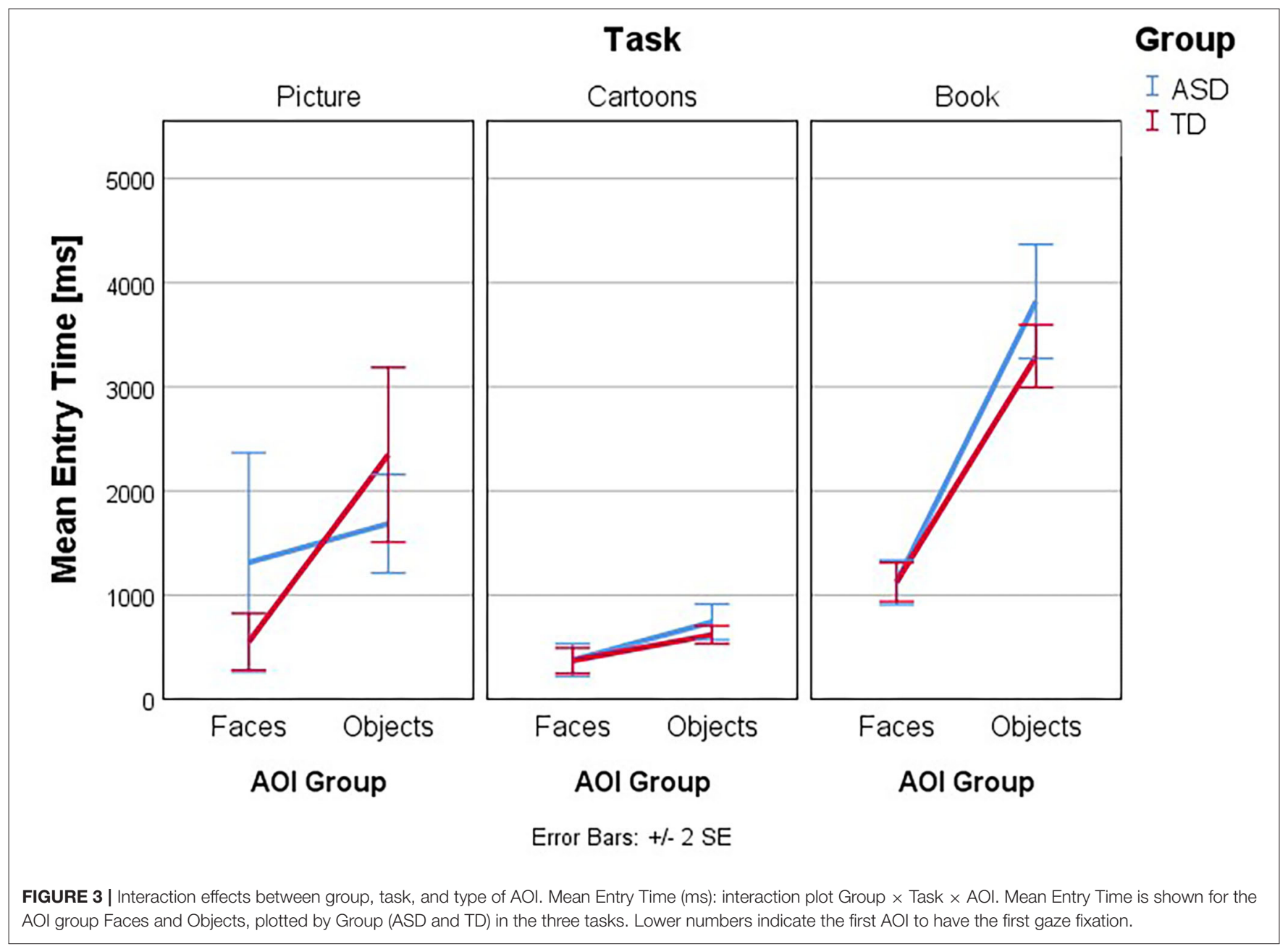




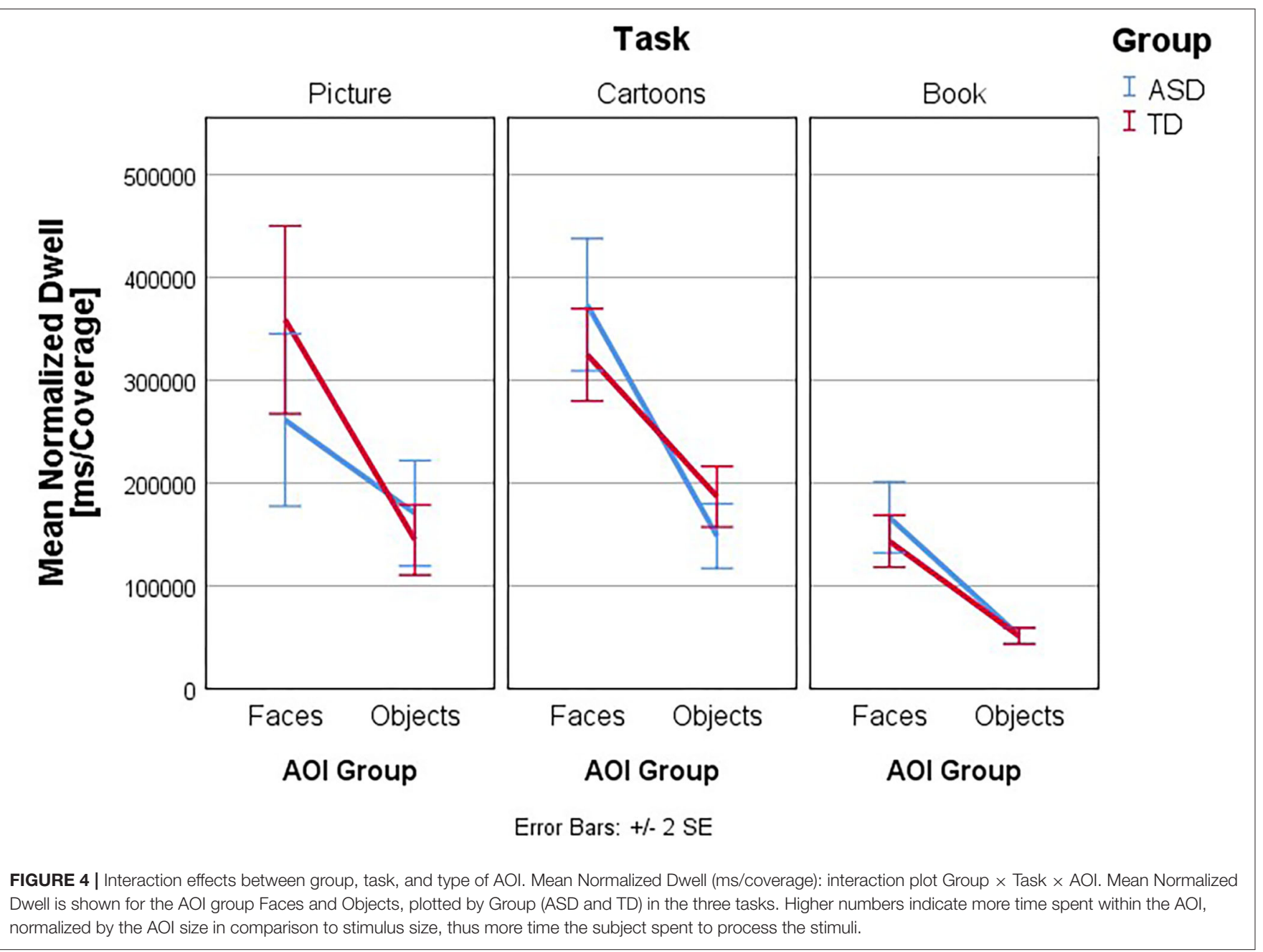

together, Pillai's Trace $=0.092 ; F_{(2,260)}=1.764, p=0.041$, partial $\eta^{2}=0.046$.

Follow-up univariate three-way ANOVAs were run for each dependent variable. These showed a statistically significant threeway interaction effect between group, task, and AOI for ET $\left[F_{(2,260)}=4.763, p=0.009\right.$, partial $\left.\eta^{2}=0.035\right], \operatorname{NormD}\left[F_{(2,260)}\right.$ $=4.805, p=0.009$, partial $\left.\eta^{2}=0.036\right], \mathrm{NDT} \%\left[F_{(2,260)}=4.693\right.$, $p=0.010$, partial $\left.\eta^{2}=0.035\right]$, and FT\% $\left[F_{(2,260)}=3.81, p=\right.$ 0.023 , partial $\left.\eta^{2}=0.029\right]$ but not for FFD $\left[F_{(2,260)}=0.59, p\right.$ $=.556$, partial $\left.\eta^{2}=0.005\right]$, Revisits $\left[F_{(2,260)}=1.77, p=0.172\right.$, partial $\left.\eta^{2}=0.013\right]$ and AFD $\left[F_{(2,260)}=0.28, p=0.756\right.$, partial $\left.\eta^{2}=0.002\right]$. The interaction effects of Group, Task, and AOI in ET and NDT\% are illustrated in Figures 3-6.

Figures 3-6 present the interaction between Task and AOI at the different groups: ASD and TD. According to Figures 3-6. This interaction effect indicates that the relationship between task and AOI depends on the group.

For the dependent variables with the statistically significant three-way interaction effect between group, task, and AOI, we now present the simple two-way interactions, main effect of group and pairwise comparisons, where needed, separately. Pairwise comparisons are summarized in Table 2.

\section{Entry Time}

Follow-up univariate two-way ANOVAs were run for the dependent variable ET and the main effect of group considered. These showed a statistically significant simple two-way interaction between Group and AOI in the dependent variable Entry Time, for the Picture Task $\left[F_{(2,260)}=9.08, p=0.003\right.$, partial $\left.\eta^{2}=0.034\right]$ but not for the Cartoons $\left[F_{(2,260)}=0.07, p=\right.$ 0.799, partial $\left.\eta^{2}=0.000\right]$ and Book $\left[F_{(2,260)}=1.27, p=0.262\right.$, partial $\left.\eta^{2}=0.005\right]$ Tasks. As such, a simple main effect analysis was conducted for Picture Task, and we found a statistically significant main effect of Group in the dependent variable Entry Time, for the AOI Faces $F_{(2,260)}=5.194, p=0.023$, partial $\eta^{2}=$ 0.020 , and for the AOI Objects $F_{(2,260)}=3.93, p=0.049$, partial $\eta^{2}=0.015$, in the Picture Task. Therefore, simple pairwise comparisons were run for the differences in mean ET score in the AOI Faces and AOI Objects between groups in the Picture Task, with a Bonferroni adjustment applied. In the Picture Task, in the AOI Faces, the mean ET in the ASD group was 1313.08 [standard deviation $(S D)=2526.63$ ] and $551.51(S D=637.78)$ in the TD group, a statistically significant mean difference of 761.56, 95\% CI $(103.55,1419.57), p=0.023, d=-0.41$. In the same task, in the AOI Objects, the mean Entry Time in 


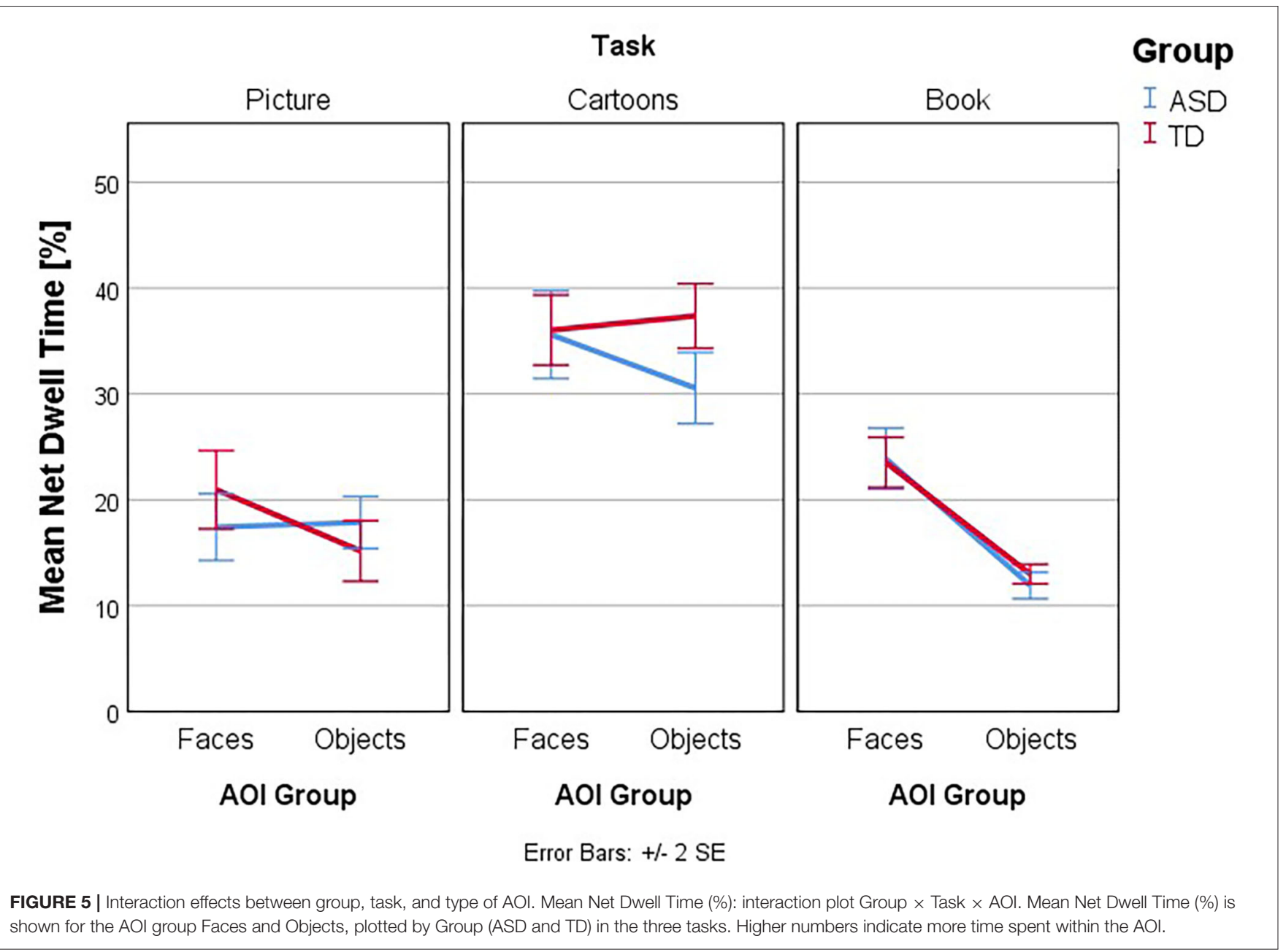

the ASD group was $1685.34(S D=1134.27)$ and 2347.37 (SD $=1967.34)$ in the TD group, a statistically significant mean difference of -662.038 , 95\% CI $(-1320.05,-4.03), p=0.049$, $d=0.41$.

\section{Normalized Dwell}

Follow-up univariate two-way ANOVAs were run for the dependent variable NormD and the main effect of group considered. These showed a statistically significant simple twoway interaction between Group and AOI in the dependent variable NormD, for the Picture task $\left[F_{(2,260)}=6.25, p=\right.$ 0.013, partial $\left.\eta^{2}=0.023\right]$ but not for the Cartoons $\left[F_{(2,260)}\right.$ $=3.17, p=0.076$, partial $\left.\eta^{2}=0.012\right]$ and Book $\left[F_{(2,260)}\right.$ $=0.21, p=0.647$, partial $\left.\eta^{2}=0.001\right]$ Tasks. Afterwards, a simple main effect analysis was conducted for Picture Task, and we found a statistically significant main effect of Group in the dependent variable NormD, for the AOI Faces $F_{(2,260)}=$ 7.79, $p=0.006$, partial $\eta^{2}=0.029$. As such, simple pairwise comparisons were run for the differences in mean NormD score in the AOI Faces and AOI Objects between groups in the Picture Task, with a Bonferroni adjustment applied. In the AOI faces in the Picture Task, the mean NormD in the ASD group was
261306.24 $(S D=200946.01)$ and $358870.71(S D=214344.38)$ in the TD group, a statistically significant mean difference of $-97564.48,95 \%$ CI $(-166398.89,-28730.07), p=0.006$, $d=0.47$.

\section{Net Dwell Time}

Follow-up univariate two-way ANOVAs were run for the dependent variable NTD\% and the main effect of group considered. These showed a statistically significant simple twoway interaction between Group and AOI in the dependent variable Net Dwell Time, for the task Picture $F_{(2,260)}=4.46, p$ $=0.036$, partial $\eta^{2}=0.017$, and for the task Cartoons $F_{(2,260)}$ $=4.80, p=0.029$, partial $\eta^{2}=0.018$ but not for the Book $\left[F_{(2,260)}=0.24, p=0.625\right.$, partial $\left.\eta^{2}=0.001\right]$ Task. As such, a simple main effect analysis was conducted for Picture and Cartoons Tasks, and we found a statistically significant main effect of Group in the dependent variable Net Dwell Time, for the AOI Faces in the Cartoons Task $F_{(2,260)}=10.97, p=$ 0.001 , partial $\eta^{2}=0.040$. Therefore, simple pairwise comparisons were run for the differences in mean NTD\% score in the AOI Faces between groups in the Cartoons Task, with a Bonferroni adjustment applied. In the AOI objects in the Cartoons Task, the 


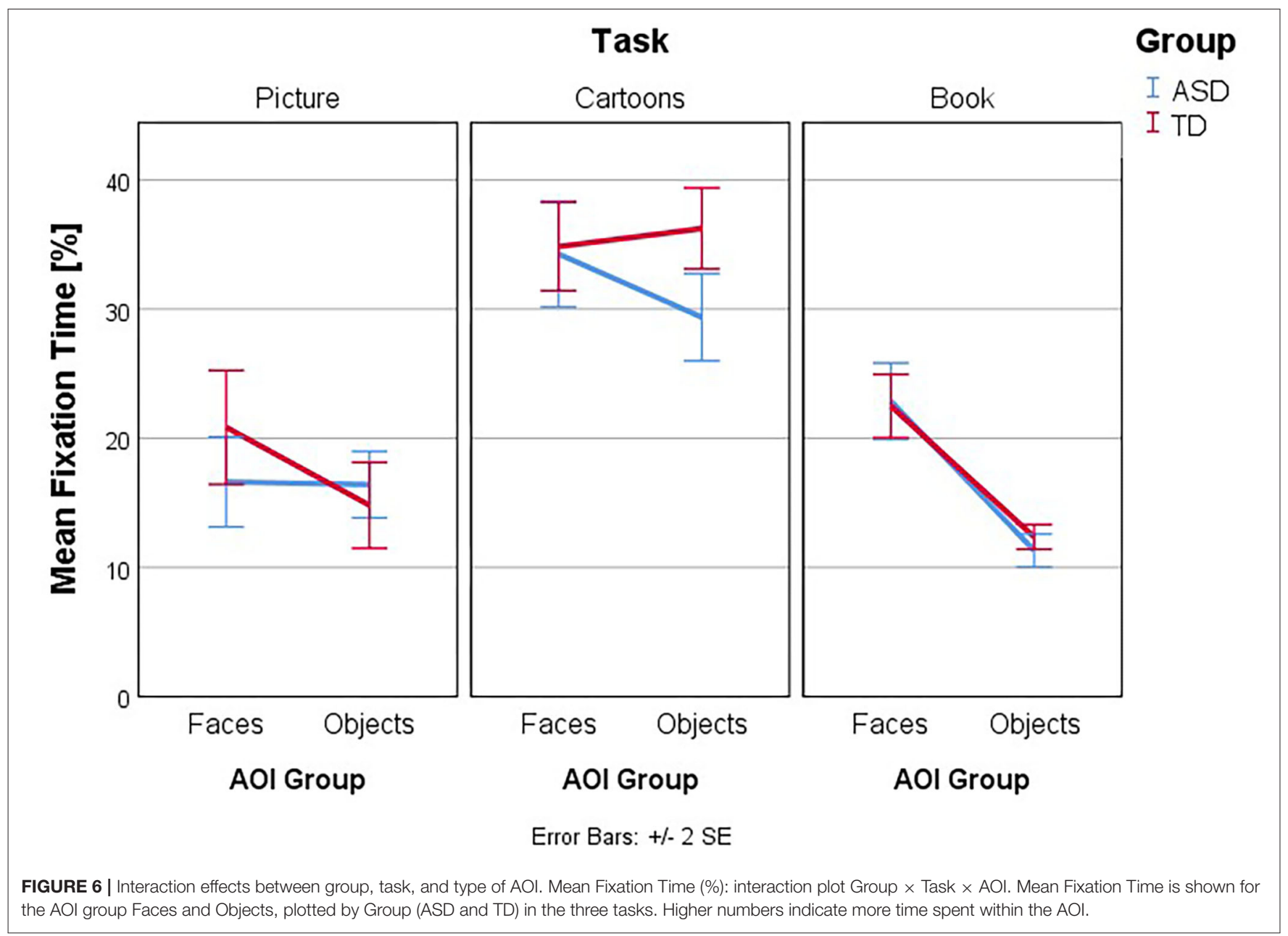

mean Net Dwell Time in the ASD group was $30.55(S D=8.06)$ and $37.37(S D=7.28)$ in the TD group, a statistically significant mean difference of $-6.82,95 \%$ CI $(-10.88,-2.77), p=0.001$, $d=0.89$.

\section{Fixation Time}

Follow-up univariate two-way ANOVAs were run for the dependent variable FT\% and the main effect of group considered. These showed a statistically significant simple twoway interaction between Group and AOI in the dependent variable $\mathrm{FT} \%$, for the task Cartoons $\left[F_{(2,260)}=4.15, p=0.043\right.$, partial $\left.\eta^{2}=0.016\right]$. Afterwards, a simple main effect analysis was conducted for Cartoons Task, and we found a statistically significant main effect of Group in the dependent variable FT\%, for the AOI Objects in the Cartoons Task $F_{(2,260)}=9.89, p=$ 0.002 , partial $\eta^{2}=0.037$. As such, simple pairwise comparisons were run for the differences in mean FT\% score in the AOI Objects between groups in the Cartoons Task, with a Bonferroni adjustment applied. In the AOI objects in the Cartoons Task, the mean Fixation Time in the ASD group was $29.36(S D=8.09)$ and $36.24(S D=7.52)$ in the TD group, a statistically significant mean difference of $-6.88,95 \%$ CI $(-11.18,-2.57), p=0.002$, $d=0.88$.

\section{DISCUSSION}

We studied social attention deficits in ASD and focused, in particular, on face stimuli, in the clinical context of different tasks of ADOS. For that purpose, we used eye-tracking methodology to compare the task dependence of visual attention to social stimuli (faces) vs. nonsocial stimuli (objects) in two matched groups of children and adolescents with ASD or TD.

We found significant interaction effects (between group, task, and type of AOI), when the participants are requested to perform spontaneous and simple descriptions of a picture or even a set of cartoons. When scenarios implied generating a goal-oriented narrative in the task, the pattern of attentional allocation in ASD subjects was normalized. In other words, it became similar to controls when children have to create a more complex story, such as the story of a book, doing an enforced narrative description. The absence of interaction effects in that case corroborates similar visual search patterns in TD children. The "Description of a Picture task," despite being a painting and not a real picture, is the one depicting a more ecological social interaction: a table surrounded by people interacting while having a lunch party, playing guitar, and talking to each other. Our findings thereby provide a framework that reconciles previous literature. Scenes 
TABLE 2 | The comparison of the mean \pm SD of the eye-tracking measures between ASD $(n=23)$ and TD $(n=23)$.

\begin{tabular}{|c|c|c|c|c|c|c|}
\hline \multirow[b]{2}{*}{$\begin{array}{l}\text { Eye-tracking } \\
\text { measures }\end{array}$} & \multirow[b]{2}{*}{ Task } & \multirow[b]{2}{*}{ AOI } & \multirow{2}{*}{$\begin{array}{c}\text { ASD } \\
\text { Mean } \pm \text { SD }\end{array}$} & \multirow{2}{*}{$\begin{array}{c}\text { TD } \\
\text { Mean } \pm \text { SD }\end{array}$} & \multicolumn{2}{|c|}{ Statistics } \\
\hline & & & & & $p$ & $d$ \\
\hline \multirow[t]{6}{*}{ Entry time (ms) } & Picture & Faces & $1313.07 \pm 2526.63$ & $551.51 \pm 637.78$ & $0.023^{\star}$ & -0.413 \\
\hline & & Objects & $1685.33 \pm 1134.27$ & $2347.37 \pm 1967.34$ & $0.049^{\star}$ & 0.412 \\
\hline & Cartoons & Faces & $375.59 \pm 375.76$ & $370.32 \pm 291.46$ & 0.987 & -0.016 \\
\hline & & Objects & $742.39 \pm 410.65$ & $617.82 \pm 207.70$ & 0.706 & -0.383 \\
\hline & Book & Faces & $1120.47 \pm 514.29$ & $1124.77 \pm 439.73$ & 0.99 & 0.009 \\
\hline & & Objects & $3821.01 \pm 1311.36$ & $3293.79 \pm 708.56$ & 0.116 & -0.497 \\
\hline \multirow{6}{*}{$\begin{array}{l}\text { Normalized dwell } \\
\text { (ms/coverage) }\end{array}$} & Picture & Faces & $261306.23 \pm 200946.10$ & $358870.71 \pm 214344.38$ & $0.006^{\star}$ & 0.47 \\
\hline & & Objects & $170705.95 \pm 122808.11$ & $144670.91 \pm 79,944.23$ & 0.457 & -0.251 \\
\hline & Cartoons & Faces & $373543.36 \pm 154277.07$ & $324851.21 \pm 107742.37$ & 0.160 & -0.366 \\
\hline & & Objects & $148538.82 \pm 75339.15$ & $186859.44 \pm 70924.83$ & 0.269 & 0.524 \\
\hline & Book & Faces & $166567.76 \pm 82569.63$ & $143424.55 \pm 59218.12$ & 0.509 & -0.321 \\
\hline & & Objects & $51705.32 \pm 19204.18$ & $51205.75 \pm 18,307.33$ & 0.989 & -0.027 \\
\hline \multirow[t]{6}{*}{ Net dwell time (\%) } & Picture & Faces & $17.42 \pm 7.55$ & $20.95 \pm 8.66$ & 0.091 & 0.435 \\
\hline & & Objects & $17.86 \pm 5.90$ & $15.16 \pm 6.70$ & 0.197 & -0.428 \\
\hline & Cartoons & Faces & $35.59 \pm 9.93$ & $36.03 \pm 7.95$ & 0.832 & 0.049 \\
\hline & & Objects & $30.55 \pm 8.06$ & $37.37 \pm 7.28$ & $0.001^{\star}$ & 0.888 \\
\hline & Book & Faces & $23.89 \pm 6.93$ & $23.53 \pm 5.54$ & 0.861 & -0.057 \\
\hline & & Objects & $11.90 \pm 3.00$ & $12.98 \pm 2.17$ & 0.605 & 0.411 \\
\hline \multirow[t]{6}{*}{ Fixation time (\%) } & Picture & Faces & $16.62 \pm 8.36$ & $20.84 \pm 10.35$ & 0.057 & 0.449 \\
\hline & & Objects & $16.41 \pm 6.16$ & $14.80 \pm 7.78$ & 0.469 & -0.229 \\
\hline & Cartoons & Faces & $34.26 \pm 9.85$ & $34.84 \pm 8.18$ & 0.792 & 0.064 \\
\hline & & Objects & $29.36 \pm 8.09$ & $36.24 \pm 7.52$ & $0.002^{\star}$ & 0.881 \\
\hline & Book & Faces & $22.87 \pm 7.07$ & $22.50 \pm 5.75$ & 0.867 & -0.057 \\
\hline & & Objects & $11.30 \pm 3.06$ & $12.35 \pm 2.25$ & 0.636 & 0.390 \\
\hline
\end{tabular}

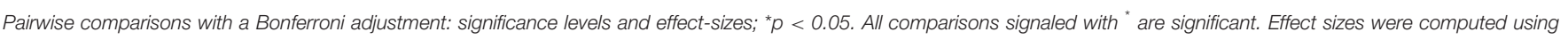
Cohen's d.

depicting ecological social interactions have been associated to better evoke robust social responses $(33,34)$. Currently, a methodological approach, denominated cognitive ethology, also focused on ecological validity. It advocates that one's research approach should begin at the level of the phenomenon of interest and to systematically move toward a simplified and abstract level but also doing the inverse approach, reinforcing the idea of analyzing a continuum $(49,50)$. In our study, we presented three different tasks, with large clinical relevance (used in ADOS) with a growing level of abstract social scenes (from the representation of the social gathering around the table, to the interaction of cartoons in the story of the book).

As predicted, we found that TD children looked first to faces and during a longer period of time in the socially rich and familiar context of a gathering of people around a table ("Description of a Picture task"). The ASD children did not show a differential pattern, between faces and objects. In other words, under these conditions interaction effects are triggered: the ASD group tends to have a similar pattern of visual search in what concerns to attention to social and non-social stimuli, that is, faces and objects, while the TD group looks first at faces and for a longer period of time, which corroborates the hypothesis that ASD participants are less attentive to faces (21-23, 36-39).
On the other hand, our study corroborates that children and adolescents with and without ASD show remarkably similar visual search patterns in their initial eye gaze to faces (51). However, in our study, participants are not instructed to specifically look at faces, which adds meaning and ecological importance to the result. In fact, the participants only had to describe what they were seeing, and therefore, the visual search pattern is natural and more spontaneous.

Overall, our results seem to provide a unifying view of previous research. The TD group always presented a lower ET to the AOI faces than objects, looking at the faces first, when exploring visually the images, also spending more time looking at social stimuli. This visual search pattern is absent in the ASD group. In fact, although children with ASD look at the faces first (lower ET in the AOI faces, than in the AOI objects), there are no statistical differences in the Cartoons and Book tasks (the ones that guide a goal oriented narrative), when compared with TD.

Taken together, our results point to the fact that social attention allocation patterns in ASD population are strongly task dependent, which extends our previous work in other cognitive domains (45). Accordingly, the task not requiring an explicit goal-oriented narrative yields the greatest differences. This raises the question whether spontaneous attention deficits can be 
rescued by guiding goal-directed actions (40). These results are relevant for the selection of interventional strategies and in ASD children, since it stresses the importance of goal-oriented actions, which are the foundations of the structured teaching.

ASD and TD groups analyze social and non-social content differently. However, when they have to do a narrative, visual behavior tends to normalize (that is, ASD has similar visual patterns as TD), which suggests that the narrative is used as guidance.

Entry time is an eye-tracking measure that characterizes rapid events, and it is in this measure that there are more differences between ASD and TD, which can be interpreted as the best measures to distinguish the groups.

In the present study, there are some limitations to consider. Inclusion of younger children and subjects with intellectual disability was difficult because most of them were not able to make a good calibration and were therefore excluded. Our experimental design did not explicitly account for low-level saliency. However, we believe that, in accordance with previous studies, gaze behavior is better predicted by the social features than by low-level saliency alone (52-54).

In the current analysis, we focused on attention to the faces and objects in the pictures from the different tasks of ADOS (a well-validated but examiner's dependent diagnosis tool for ASD) thereby trying to provide a complementary quantitative information of potential value in clinical practice to distinguish between ASD children without intellectual disability and TD. Although precise, the sensitivity of eye-tracking as a diagnostic tool remains uncertain. Here, we provide evidence for task dependence, with patterns "normalizing" when a narrative is required. With this strategy, we hope to provide a tool that may help improve the course of ASD diagnostic evaluation, especially in subjects with ASD without intellectual disability, where the differential diagnosis with a typical neurodevelopment is often very difficult.

In conclusion, eye-tracking measures of visual scanning, while exploring and describing activities from the ADOS, in particular, "Description of a Picture," can discriminate between ASD and TD groups. Individuals with ASD allocated their attention to faces and objects in a similar way, while individuals from the TD group attend first and more time to faces. However, when ASD children are asked to look at pictures, organize the thought, and tell a story, they attend to the same stimuli and have a similar pattern of visual search as the TD group, which raises interesting insights on the origin of this "normalization." Accordingly, when goaldirected actions, in this case, an enforced narrative description, are required, visual search patterns in ASD tend to resemble TD and therefore "normalize" as compared to spontaneous attention. These findings are of potential relevance to training strategies, by

\section{REFERENCES}

1. American Psychiatric Association. Diagnostic and Statistical Manual of Mental Disorders. 5th ed. Arlington, VA: American Psychiatric Publishing (2013). doi: 10.1176/appi.books.97808904 25596 providing clues on learning adaptive attentional deployment. In addition, they stress the importance from a diagnostic perspective point of view of observation and classification of spontaneous behavior. Future work should confirm the value of this tool to help differential diagnosis especially in difficult cases with other neurodevelopmental disorders or typical development.

\section{DATA AVAILABILITY STATEMENT}

The raw data supporting the conclusions of this article will be made available by the authors, without undue reservation.

\section{ETHICS STATEMENT}

All procedures in this study were reviewed and approved by the ethics committees from the Faculty of Medicine from University of Coimbra, Portugal (CE-11/2013) and the Centro Hospitalar e Universitário de Coimbra, Portugal (CHUC-102-13) and was conducted in accordance with the 1964 Helsinki declaration and its later amendments or comparable ethical standards. Written informed consent was obtained from the parents/guardians of all participants. Children and adolescents also gave oral informed consent.

\section{AUTHOR CONTRIBUTIONS}

SM, GO, and MC-B: conceived and designed the study. SM: performed the study and wrote the original manuscript. SM, CC, DS, and FD: contributed with data collection. SM and JC: analyzed the data. GO and MC-B: supervision. JC, GO, and MC-B: reviewed and edited the manuscript. All authors read and approved the final manuscript.

\section{FUNDING}

This research was supported by the Portuguese Foundation for Science and Technology: PAC MEDPERSYST POCI-01-0145FEDER-016428, CONNECT-BCI, POCI-01-0145-FEDER-30852, FCT/UID/4950/2020, DSAIPA/DS/0041/2020, individual Ph.D. scholarship: SFRH/BD/102779/2014 to SM, CENTRO-01-0145FEDER-000016 to SM and DS (PI: MC-B), and Luso-American Development Foundation (FLAD) Life Sciences. The funders had no role in study design, data collection and analysis, decision to publish, or preparation of the manuscript.

\section{ACKNOWLEDGMENTS}

The authors thank all participants and their families for their willingness to participate in this study, particularly the participants with autism spectrum disorder.

2. Chita-Tegmark M. Social attention in ASD: a review and meta-analysis of eye-tracking studies. Res Dev Disabil. (2016) 48:79-93. doi: 10.1016/j.ridd.2015.10.011

3. Chita-Tegmark M. Attention allocation in ASD: a review and metaanalysis of eye-tracking studies. Rev J Autism Dev Disord. (2016) 3:20923. doi: $10.1007 / \mathrm{s} 40489-016-0077-\mathrm{x}$ 
4. Falck-Ytter T, Bölte S, Gredebäck G. Eye tracking in early autism research. $J$ Neurodev Disord. (2013) 5:28. doi: 10.1186/1866-1955-5-28

5. Guillon Q, Hadjikhani N, Baduel S, Rogé B. Visual social attention in autism spectrum disorder: insights from eye tracking studies. Neurosci Biobehav Rev. (2014) 42:279-97. doi: 10.1016/j.neubiorev.2014.03.013

6. Gliga T, Csibra G. Seeing the face through the eyes: a developmental perspective on face expertise. In: von Hofsten C, Rosander K, editors. Progress in Brain Research. Vol. 164. Amsterdam: Elsevier (2007). p. 323-39. doi: 10.1016/S0079-6123(07)64018-7

7. Goren CC, Sarty M, Wu PYK. Visual following and pattern discrimination of face-like stimuli by newborn infants. Pediatrics. (1975) 56:544-9.

8. Vuilleumier P. Facial expression and selective attention. Curr Opin Psychiatry. (2002) 15:291-300. doi: 10.1097/00001504-200205000-00011

9. Feldman R, Greenbaum CW, Yirmiya N. Mother-infant affect synchrony as an antecedent of the emergence of self-control. Dev Psychol. (1999) 35:22331. doi: $10.1037 / 0012-1649.35 .1 .223$

10. Tronick EZ. Emotions and emotional communication in infants. Am Psychol. (1989) 44:112-9. doi: 10.1037/0003-066X.44.2.112

11. Trevarthen C, Aitken KJ. Infant intersubjectivity: research, theory, and clinical applications. J Child Psychol Psychiatry. (2001) 42:3-48. doi: 10.1111/1469-7610.00701

12. Grelotti DJ, Gauthier I, Schultz RT. Social interest and the development of cortical face specialization: what autism teaches us about face processing. Dev Psychobiol. (2002) 40:213-25. doi: 10.1002/dev.10028

13. Johnson MH. Subcortical face processing. Nat Rev Neurosci. (2005) 6:76674. doi: $10.1038 / \mathrm{nrn} 1766$

14. Schultz RT. Developmental deficits in social perception in autism: the role of the amygdala and fusiform face area. Int J Dev Neurosci. (2005) 23:12541. doi: 10.1016/j.ijdevneu.2004.12.012

15. Chevallier C, Kohls G, Troiani V, Brodkin ES, Schultz RT. The social motivation theory of autism. Trends Cogn Sci. (2012) 16:231-9. doi: 10.1016/j.tics.2012.02.007

16. Dawson G, Webb SJ, McPartland J. Understanding the nature of face processing impairment in autism: insights from behavioral and electrophysiological studies. Dev Neuropsychol. (2005) 27:403-24. doi: 10.1207/s15326942dn2703_6

17. Pelphrey KA, Sasson NJ, Reznick JS, Paul G, Goldman BD, Piven J. Visual scanning of faces in autism. J Autism Dev Disord. (2002) 32:24961. doi: 10.1023/A:1016374617369

18. Wagner JB, Hirsch SB, Vogel-Farley VK, Redcay E, Nelson CA. Eye-tracking, autonomic, and electrophysiological correlates of emotional face processing in adolescents with autism spectrum disorder. J Autism Dev Disord. (2013) 43:188-99. doi: 10.1007/s10803-012-1565-1

19. Bird G, Press C, Richardson DC. The role of alexithymia in reduced eyefixation in autism spectrum conditions. J Autism Dev Disord. (2011) 41:155664. doi: 10.1007/s10803-011-1183-3

20. Chawarska K, MacAri S, Shic F. Context modulates attention to social scenes in toddlers with autism. J Child Psychol Psychiatry Allied Discip. (2012) 53:903-13. doi: 10.1111/j.1469-7610.2012.02538.x

21. Klin A, Jones W, Schultz R, Volkmar F, Cohen D. Visual fixation patterns during viewing of naturalistic social situations as predictors of social competence in individuals with autism. Arch Gen Psychiatry. (2002) 59:80916. doi: 10.1001/archpsyc.59.9.809

22. Shic F, Bradshaw J, Klin A, Scassellati B, Chawarska K. Limited activity monitoring in toddlers with autism spectrum disorder. Brain Res. (2011) 1380:246-54. doi: 10.1016/j.brainres.2010.11.074

23. Riby DM, Hancock PJB. Do faces capture the attention of individuals with williams syndrome or autism? Evidence from tracking eye movements. J Autism Dev Disord. (2009) 39:421-31. doi: 10.1007/s10803-0080641-z

24. Wilson CE, Brock J, Palermo R. Attention to social stimuli and facial identity recognition skills in autism spectrum disorder. J Intellect Disabil Res. (2010) 54:1104-15. doi: 10.1111/j.1365-2788.2010.01340.x

25. Pierce K, Marinero S, Hazin R, McKenna B, Barnes CC, Malige A. Eye tracking reveals abnormal visual preference for geometric images as an early biomarker of an autism spectrum disorder subtype associated with increased symptom severity. Biol Psychiatry. (2016) 79:657-66. doi: 10.1016/j.biopsych.2015.03.032
26. Frazier TW, Strauss M, Klingemier EW, Zetzer EE, Hardan AY, Eng C, et al. A meta-analysis of gaze differences to social and nonsocial information between individuals with and without autism. J Am Acad Child Adolesc Psychiatry. (2017) 56:546-55. doi: 10.1016/j.jaac.2017.05.005

27. Kemner C, van der Geest JN, Verbaten MN, van Engeland $H$. Effects of object complexity and type on the gaze behavior of children with pervasive developmental disorder. Brain Cogn. (2007) 65:107-11. doi: 10.1016/j.bandc.2006.05.006

28. Parish-Morris J, Chevallier C, Tonge N, Letzen J, Pandey J, Schultz RT. Visual attention to dynamic faces and objects is linked to face processing skills: a combined study of children with autism and controls. Front Psychol. (2013) 4:1-7. doi: 10.3389/fpsyg.2013.00185

29. Kuhn G, Kourkoulou A, Leekam SR. How magic changes our expectations about autism. Psychol Sci. (2010) 21:1487-3. doi: 10.1177/0956797610383435

30. Di Giorgio E, Frasnelli E, Rosa Salva O, Scattoni ML, Puopolo M, Tosoni D, et al. Difference in visual social predispositions between newborns at low- and high-risk for autism. Sci Rep. (2016) 6:26395. doi: 10.1038/srep29860

31. Elsabbagh M, Gliga T, Pickles A, Hudry K, Charman T, Johnson MH. The development of face orienting mechanisms in infants at-risk for autism. Behav Brain Res. (2013) 251:147-54. doi: 10.1016/j.bbr.2012.07.030

32. Macari S, Milgramm A, Reed J, Shic F, Powell KK, Macris D, et al. Contextspecific dyadic attention vulnerabilities during the first year in infants later developing autism spectrum disorder. J Am Acad Child Adolesc Psychiatry. (2021) 60:166-75. doi: 10.1016/j.jaac.2019.12.012

33. Falck-Ytter T, von Hofsten C. How special is social looking in ASD. In: Braddick JAO, Innocenti G editors, Progress in Brain Research. Elsevier B.V (2011). p. 209-222. doi: 10.1016/B978-0-444-53884-0.00026-9

34. Saitovitch A, Bargiacchi A, Chabane N, Phillipe A, Brunelle F, Boddaert N, et al. Studying gaze abnormalities in autism: which type of stimulus to use? Open J Psychiatry. (2013) 3:32-8. doi: 10.4236/ojpsych.2013.32A006

35. Chevallier C, Parish-Morris J, Mcvey A, Rump KM, Sasson NJ, Herrington JD, et al. Measuring social attention and motivation in autism spectrum disorder using eye-tracking: stimulus type matters. Autism Res. (2015) 8:6208. doi: 10.1002/aur.1479

36. Rice K, Moriuchi JM, Jones W, Klin A. Parsing heterogeneity in autism spectrum disorders: visual scanning of dynamic social scenes in schoolaged children. J Am Acad Child Adolesc Psychiatry. (2012) 51:23848. doi: 10.1016/j.jaac.2011.12.017

37. Kirchner JC, Hatri A, Heekeren HR, Dziobek I. Autistic symptomatology, face processing abilities, and eye fixation patterns. J Autism Dev Disord. (2011) 41:158-67. doi: 10.1007/s10803-010-1032-9

38. Riby DM, Hancock PJ, Jones N, Hanley M. Spontaneous and cued gazefollowing in autism and Williams syndrome. J Neurodev Disord. (2013) 5:13. doi: $10.1186 / 1866-1955-5-13$

39. Shi L, Zhou Y, Ou J, Gong J, Wang S, Cui X, et al. Different visual preference patterns in response to simple and complex dynamic social stimuli in preschool-aged children with autism spectrum disorders. PLoS ONE. (2015) 10:e0122280. doi: 10.1371/journal.pone.0122280

40. Birmingham E, Cerf M, Adolphs R. Comparing social attention in autism and amygdala lesions: effects of stimulus and task condition. Soc Neurosci. (2011) 6:420-435. doi: 10.1080/17470919.2011.561547

41. Freeth M, Ropar D, Chapman P, Mitchell P. The eye gaze direction of an observed person can bias perception, memory, and attention in adolescents with and without autism spectrum disorder. $J$ Exp Child Psychol. (2010) 105:20-37. doi: 10.1016/j.jecp.2009. 10.001

42. Freeth M, Ropar D, Mitchell P, Chapman P, Loher S. Brief report: how adolescents with ASD process social information in complex scenes. Combining evidence from eye movements and verbal descriptions. J Autism Dev Disord. (2011) 41:364-71. doi: 10.1007/s10803-0101053-4

43. Van Der Geest JN, Kemner C, Camfferman G, Verbaten MN, Van Engeland $\mathrm{H}$. Looking at images with human figures: comparison between autistic and normal children. J Autism Dev Disord. (2002) 32:6975. doi: 10.1023/A:1014832420206

44. Marsh LE, Pearson A, Ropar D, de Hamilton CAF. Predictive gaze during observation of irrational actions in adults with autism spectrum conditions. J Autism Dev Disord. (2015) 45:245-61. doi: 10.1007/s10803-014-2215-6 
45. Bernardino I, Mouga S, Almeida J, van Asselen M, Oliveira G, CasteloBranco M. A direct comparison of local-global integration in autism and other developmental disorders: implications for the central coherence hypothesis. PLoS ONE. (2012) 7:e39351. doi: 10.1371/journal.pone.0 039351

46. Lord C, Rutter M. Autism Diagnostic Observation Schedule-WPS (ADOSWPS). Los Angeles, CA: West Psychol. (1999). doi: 10.1037/t17256-000

47. Jarrold C, Brock J. To match or not to match? Methodological issues in autism-related research. J Autism Dev Disord. (2004) 34:81-6. doi: 10.1023/B:JADD.0000018078.82542.ab

48. Le Couteur A, Lord C, Rutter M. The Autism Diagnostic Interview-Revised (ADI-R). Los Angeles, CA: Western Psychological Services. (2003).

49. Risko EF, Laidlaw KEW, Freeth M, Foulsham T, Kingstone A. Social attention with real versus reel stimuli: toward an empirical approach to concerns about ecological validity. Front Hum Neurosci. (2012) 6:111. doi: 10.3389/fnhum. 2012.00143

50. Kingstone A. Taking a real look at social attention. Curr Opin Neurobiol. (2009) 19:52-6. doi: 10.1016/j.conb.2009.05.004

51. Schauder KB, Park WJ, Tsank Y, Eckstein MP, Tadin D, Bennetto L. Initial eye gaze to faces and its functional consequence on face identification abilities in autism spectrum disorder. J Neurodev Disord. (2019) 11:120. doi: 10.1186/s11689-019-9303-Z
52. Birmingham E, Bischof WF, Kingstone A. Saliency does not account for fixations to eyes within social scenes. Vision Res. (2009) 49:29923000. doi: 10.1016/j.visres.2009.09.014

53. End A, Gamer M. Preferential processing of social features and their interplay with physical saliency in complex naturalistic scenes. Front Psychol. (2017) 8:1-16. doi: 10.3389/fpsyg.2017.00418

54. Fletcher-Watson S, Leekam SR, Benson V, Frank MC, Findlay JM. Eye-movements reveal attention to social information in autism spectrum disorder. Neuropsychologia. (2009) 47:24857. doi: 10.1016/j.neuropsychologia.2008.07.016

Conflict of Interest: The authors declare that the research was conducted in the absence of any commercial or financial relationships that could be construed as a potential conflict of interest.

Copyright (c) 2021 Mouga, Castelhano, Café, Sousa, Duque, Oliveira and CasteloBranco. This is an open-access article distributed under the terms of the Creative Commons Attribution License (CC BY). The use, distribution or reproduction in other forums is permitted, provided the original author(s) and the copyright owner(s) are credited and that the original publication in this journal is cited, in accordance with accepted academic practice. No use, distribution or reproduction is permitted which does not comply with these terms. 